\title{
TRAO ĐÖI
}

LTS: Mục TRAO DỒ của tạp chi Khoa học tính tón và điêu khiên sê lần lượt giới thiệu các kinh nghięm của đông đảo bạn đọc về những vã̃n đề sau đây :

- Các kỷ thuật lập trình

- Các tồ chức dữ liệu

- Các thuật toán viết bằng ngôn ngữ lập trinh

- Những vấn đề của kỹ thuật xứ lý (phần cứng)

- Thiết kế và các cài đặt các hệ tin bọc

- Va tất cả những họ̣t động liên quan tới sự phát triên của khoa học tính toán và điều khièn.

Rất mong aược bạn đọc xa găn gửi tới tỏa soạn những kinh nghię̣m thu aược trong hoạt động tin học, những ł kiến nhận xêt, đề nghị cải tiển những nọi dung đã cơng bố...

\section{CHUYỀ ĐÔI MỌT CHƯơNG TRINH PASCAL SANG CHUOONG TRINH BASIC}

1- Dột vắn ảa : Ngon ngữ lập trình PASCAL và phương án mớ rộng PASCAL MODULA do Niklaux Wirth đề xuất ngày nay dã được cài đặt trên hầu hết các loại máy tính, từ.maxi (super), macro đến mini, micro. Các chương trình mẫu vậa dụng những kỹ thuật lập trinh cao cấp thao tác trên các kiều dữ kiện đủ mạnh được công bố tiên nhiêu tập chí và các sách tin học kinh điên. Tháng 10 năm 1984, tác giả của ngôn ngũ̃ PASCAL đã được trao tạng giải thưỏng Tưing về những đóng góp giá trị cho kỹ thuật tính toán. ở nước ta. PASCAL MODULA và các phương án PASCAL MTPLUS, UCSD được cài đặl trên một số máy vi tính 8 và 16 bit.

Ngôn ngữ lạ̣p trình BASIC cài đặt trên các loại máy vi tính và mini hiện đang được phờ biên rọng rãi tại nhiếu cơ quan nghiên cứu và sản xuất cịa nước ta.

Trong bài nay sẽ giới thię̂u cách chuyên mọt chương trình PASCAL sang chương trình BASIC nhằm tận dụng the mạnh của bai ngơn ngữ nơi trên.

\section{II - Tài lị̣̂u và mழ̣t 36 quy ức:}

Chương trinh PASCAL sè đượ viết theo phương án trong tải liệu a Pascal User Manuel and Report s (2nd ed., 1976) của K. Jensen và N. Wirth, chương trinh BASIC (APPLESOFT Iİ) đưgc viêt theo phương án trong tài liệu « Phăn mềm hẹ thống APPLEII n (Vũ Duy Mẳn bièn dịch, 1984).

Bài viết sẽ sử dụng các kł hiệu sau dâ :

P. P1, P2,... mă biêu cho các doạn cong trinh PASCAL

B. B1, B2,... mä biệu cho céc doun trinh BASIC

$t, t 1, t_{2}, . ., k y$ hię̣u cho các câu lę̣nh

$\mathrm{dk}$, đk1, đk2,... ký hiẹu cho cár bièu thức logic nhận giá trị đúng hoạc sai trong các câu lę̣nh cồ điều kiện

bt, bt1, bt2,... ký hiệu cho cáe biêu thức sờ hoạc lógic

$N, N 1, N 2, \ldots$ ký bięu só dòng troñg chưag trinh BASIC

a.A,b,B.x,X... ký hiện cho cáe bién.

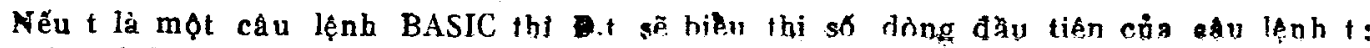

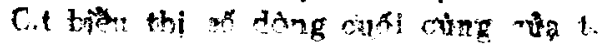


III - Sự twơng đương giữa các Iệnh PA5CAL và BAsIC:

1. Lệnh gán

(P1) $x:=b t$

(B1) $N$ LET $X=b t$

hoặc (B1.1) $\mathrm{Nx}=\mathrm{bt}$

2. Lệnh diêu kiện

(P2) IF dk THEN t

(B2) N IF đk THEN t.

Nểu câu lệnh $t$ phải viết trải trên nhiều dơng, từ dòng Đ.t đễn dong C.t chẳng hạn thi dùng đoạn trinh (B2.1) sau đây :

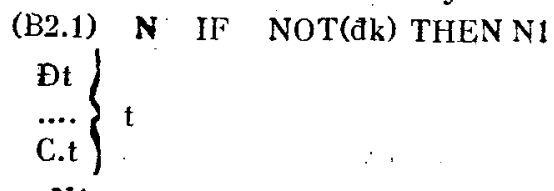

N1...

(P3) IF ak THFN t1 ELSE $t 2$.

Trong BASIC (APPLESOFT II) không có từ khóa ELSE. ta chuyễn (P3) thành (B3) nbư sau (B3) $N$ IF NOT(dk) THEN Đt2
Đ. $\mathbf{t 1}$
C..$\dot{t 1}\} t$
N1 GOTO N2
$Ð$. 12
$\because \cdots t 2$
N2

Những đoan trình (B3.1) - (B3.3) dưới dây dều tương đương với (B3) và do đó chúng tương đương với (P3). Việc dùng đọnn trình nào phụ thuộc vào chiều dài của dãy lệnh t1 và t2 (các lệnh đó có thè viết trên 1 dòng hay không) và mức độ phức tạp của biều thức điều kiện đk.
(B3.1)

$$
\begin{aligned}
& \left.\begin{array}{l}
\text { IF dk THE } \\
\text { Đ. } t 2 \\
\text { C. } \cdot t_{2}
\end{array}\right\} t 2 \\
& \text { N1 GOTO N2 } \\
& \left.\begin{array}{l} 
\pm . t 1 \\
\text { C. } . t 1
\end{array}\right\} t 1
\end{aligned}
$$$$
\text { N2... }
$$

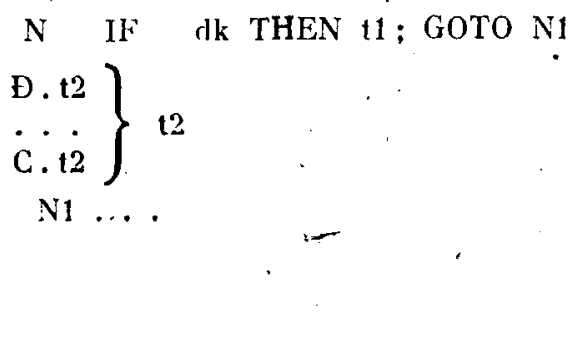

(B3.3)

N IF NOT(ak) THEN t2:GOTO N1

$$
\left.\begin{array}{l}
\boxplus \cdot t 1 \\
\text { C. }: t 1
\end{array}\right\} t
$$

3. Lệnh lặp với điều kiện trước

(P4) WHILE dk DO $t$

(B4)

$$
\begin{aligned}
& \text { N IF NOT(dk) THEN N2 } \\
& \left.\begin{array}{c}
\text { D.t } \\
\text { C. } . t
\end{array}\right\} t \\
& \text { N GOTO N }
\end{aligned}
$$

N2.. 
4. Lệnh lặ với diều klện sau

(P5) REPEAT $t$ UNTIL $d k$

(B5)

$$
\left.\begin{array}{l}
\text { D.t } \\
\text { C. . }
\end{array}\right\} t
$$

N1 IF NOT (dk) THEN Đ.t

5. Lệnh lặ theo tham biễn

(P6) FOR $\mathrm{i}:=\mathrm{bt} 1$ TO bt2 DO $t$

(B6)

$\mathrm{N} \quad \mathrm{I}=\mathrm{bt} 1: \mathrm{K}=\mathrm{bt} 2$

$\begin{array}{lllll}\text { N1 } & \text { IF } & \text { I }>K & \text { THEN } & \text { N3 }\end{array}$

$$
\left.\begin{array}{l}
\text { घ.t } \\
\mathrm{C} . \mathrm{t}
\end{array}\right\} t
$$

N2 I $=\mathrm{I}+1:$ GOTO N1

$\mathrm{N} 3 \ldots$

(P7) FOR i : $=$ bt1 B̈́WNTO bt2 DO 1

(B7) N I $=\mathrm{bt1}: \mathrm{K}=\mathrm{bt2}$

$\mathrm{N} 1 \mathrm{IF} \quad \mathrm{I}<\mathrm{K}$ THEN N3

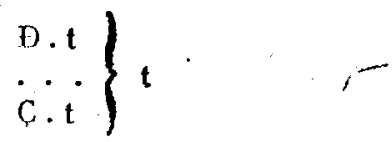

N2, I $=\mathrm{I}-1$ : GOTO N1

N3 ...

Vi lệnh lặp theo tham biến của BASIC thực hiện kiềm tra điều kiện sau (và do đó lệnh $t$ được thực hiện it ra là một lần) trong khi lệnh lặp theo tham biến của PASCAL thực hiện kiềm tra điều kiện trước nên đoạn trình (B8) sẽ tương đương với (P8) sau đây :

\section{(B8)}

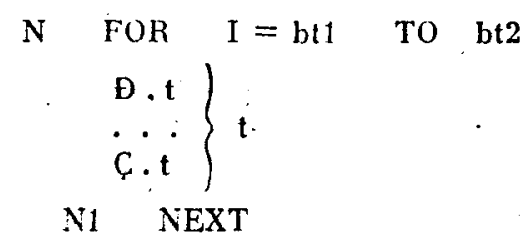

Tương tự ta có (B9) tượg aương rới (P9) nếu bt $3>0$ và tương đương với (P9, $\mathrm{A})$

(B9)

N FOR I - bt1 TO bł2 STEP bts

$\left.\begin{array}{l}\text { A.t } \\ \text { C. } t\end{array}\right\}$

N1 NEXT

(PR) $\mathrm{i}:=\mathrm{bt} 1 ; \mathrm{k}:=\mathrm{bt} 2 ; \mathrm{b}:=\mathrm{bt} 3$

FEPEAT

t;

$\mathrm{i}: \equiv \mathrm{i}+\mathrm{h}$

UNTIL $(i \geqslant k)$

(P0.A) $\mathrm{i}:=\mathrm{bt} 1 ; \mathrm{k}:=\mathrm{bt} 2 ; \mathrm{h}:=\mathrm{bt} 3$

REPEAT

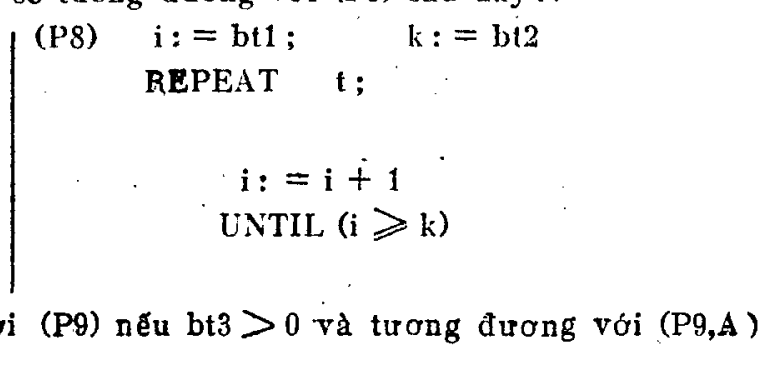

$$
\mathbf{i}:=\mathbf{i}+\mathbf{1}
$$




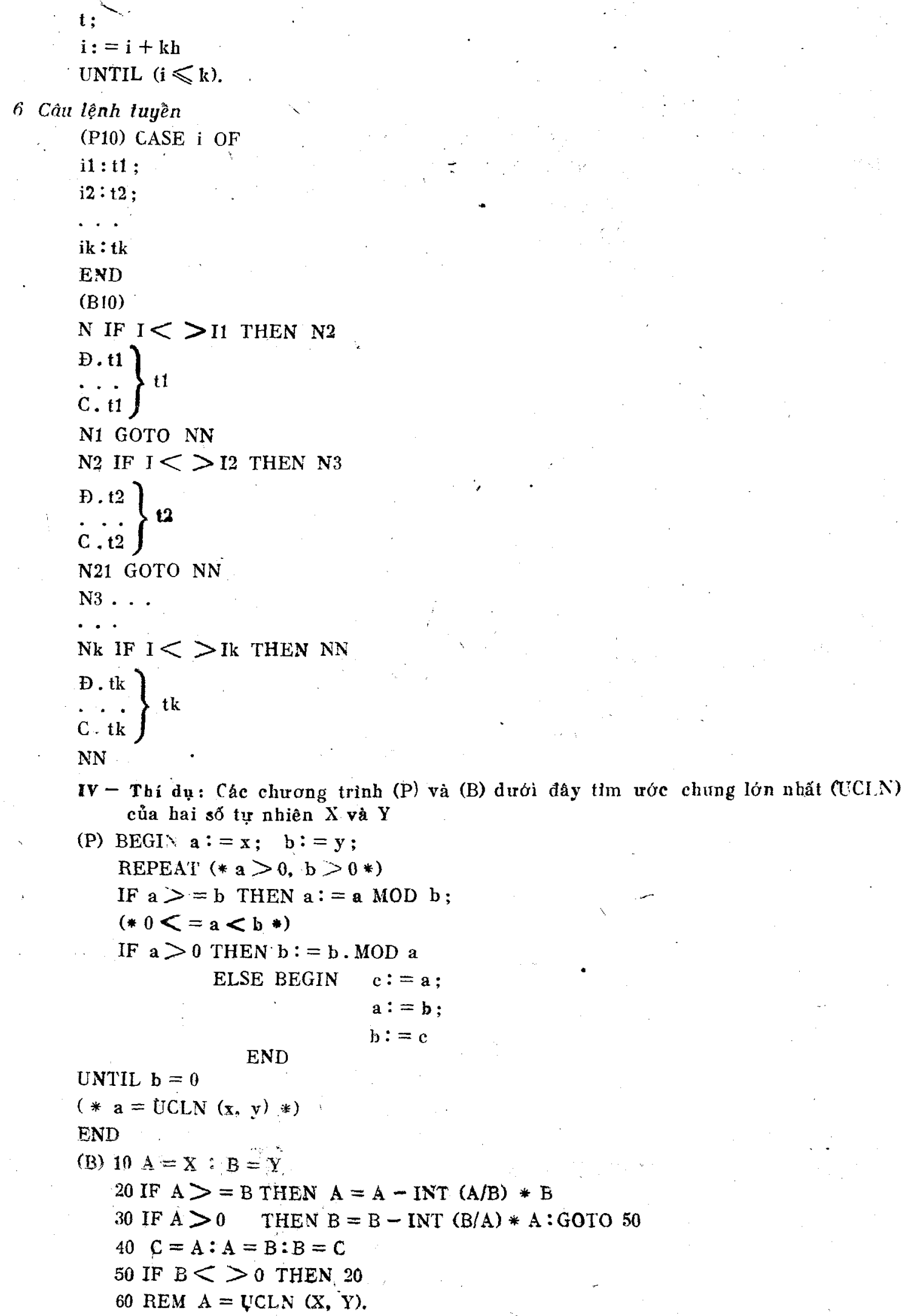

